\section{The future of melanoma therapy: developing new drugs and improving the use of old ones}

\author{
“...while most of the studies involve new drugs, it could be helpful to \\ have a small number of clinical trials investigating the best way to use \\ the already available treatments. In this way, we could have an accurate \\ algorithm and guidelines, helping both patients and practitioners, in \\ few years."
}

\section{Giuliano Palumbo ${ }^{*, 1}$, Giuseppe Di Lorenzo', Margaret Ottaviano' \& Vincenzo Damiano'}

First draft submitted: 1 December 2015; Accepted for publication: 29 June 2016; Published online: 7 October 2016

The treatment of metastatic melanomas is one of the most interesting and exciting fields in oncology. In fact, worldwide, seven drugs, including three therapeutic immune checkpoint inhibitors [1] and four BRAFMEK blockers [2], have been approved for use since 2011.

The interaction between the immune system and the melanoma has always been one of the peculiarities of this disease. The first attempt to develop a treatment that could improve the immunological activity against melanoma, through IL-2, provided encouraging results in terms of activity; however, the associated toxicity immediately discouraged routine use [3].

In 2010, the advent of ipilimumab, an anti-CTLA- 4 inhibitor, changed the whole scenario. It demonstrated an outstanding median overall survival (OS) rate in 10 months; however, it did not offer an advantage in progression-free survival (PFS) [4].

In the following years, many studies confirmed the efficacy of nivolumab [5] and pembrolizumab [6], two PD-1 inhibitors, as a first-line treatment. Both significantly improved the OS rate, with similar toxicity profiles.
In the beginning of 2015, a new study combining ipilimumab and nivolumab showed an outstanding improvement in median PFS (11.5 months against 2.9 months with ipilimumab), although $36.4 \%$ of patients interrupted the treatment owing to grade 3-4 toxicities and side effects, mainly diarrhea and colitis [7].

On the target therapy side, the MAPK/ ERK pathway was investigated and a target was found in BRAF V600-mutated melanoma. Vemurafenib [8] and dabrafenib [9] are two selective BRAF inhibitors that were approved in 2011 and 2013, respectively. Both were compared with dacarbazine and showed a median PFS of about 5 months.

Target therapies are usually confounded by the appearance of resistance mechanisms, and BRAF inhibitors are no exception. The MEK protein, a kinase collocated downstream from the BRAF protein in the MAPK/ERK pathway, seemed to be a promising target for a therapeutic agent that could improve the results achieved with BRAF inhibitors.

Two MEK inhibitors, trametinib and cobimetinib, were introduced. Two studies combining a BRAF inhibitor and a MEK inhibitor (dabrafenib plus trametinib vs

'Medical Oncology Unit, Department of Clinical Medicine, Federico II University, 80138 Naples, Italy

*Author for correspondence: Tel.: +39 081 7463660; Fax: +39 081 2203147; giuliano.palumbo@yahoo.it

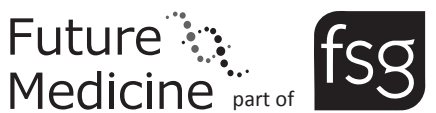

\section{KEYWORDS}

- adjuvant treatment $\bullet$ adoptive cell therapy $\bullet$ biological therapy • checkpoint inhibitors

- immunotherapy • ipilimumab

- MEK inhibitor $\bullet$ melanoma $\bullet$ target therapy BRAF inhibitor
"The treatment of metastatic melanomas is one of the most interesting and exciting fields in oncology." 
"Although there are a great number of new molecules, with a wide spectrum of clinical studies, it would also be interesting to understand how the already approved drugs could be combined for treatment or which drug is best suited to first-line treatment of BRAF-mutated melanomas." only dabrafenib and vemurafenib plus cobimetinib vs only vemurafenib) demonstrated OS and PFS improvements in combination (median OS in the dabrafenib plus trametinib study was 25.2 months against 18.7 with dabrafenib only; the median PFS with the vemurafenib plus cobimetinib combination was 9.9 months against 6.2 months with vemurafenib only) with a reduction of adverse events [10,11].

In the last few years, outstanding results in melanoma treatment have been seen; however, further progress can be made through discovering new pathways and treatments and improving the use of readily available drugs.

\section{Adjuvant therapy}

Before October 2015, there was only one adjuvant option available for high-risk patients, which was IFN- $\alpha$. Administered as recommended by Kirkwood et al., it significantly reduces the risk of recurrence [12]; however, the mechanism of action is still not fully understood and there are many side effects, including longterm ones. In fact, with the advent of new drugs, many are questioning the utility of the IFN- $\alpha$ in the adjuvant setting.

Ipilimumab has been adopted in the USA as an adjuvant drug, and probably will soon be used worldwide in the adjuvant setting. The EORTC 18071 study found that ipilimumab improves the median recurrence-free survival to $25 \%$ against the placebo, $17.1 \%$, in stage III resected melanoma [13]. However, the high percentage of grade 3-5 adverse events (such as colitis, diarrhea and thyroiditis) and the higher-than-average dosage $(10 \mathrm{mg} / \mathrm{kg})$, led to the necessity of a new trial, the ECOG 1609. This study will compare the administration of ipilimumab at $10 \mathrm{mg} / \mathrm{kg}$ dose versus the administration of ipilimumab at $3 \mathrm{mg} / \mathrm{kg}$ dose versus high-dose IFN- $\alpha$, using OS and recurrence-free survival as the primary end points. Its results are expected in 2019, and will highlight the best dosage. Furthermore, it will provide a direct comparison between ipilimumab and interferon in the adjuvant setting.

On the target therapy side, both the MEK and BRAF combinations, dabrafenib plus trametinib and vemurafenib plus cobimetinib, are being evaluated in ongoing studies, a Phase III (COMBI-AD) and a Phase II trial, respectively, both in stage III melanomas after surgery. The first data from the COMBI-AD trial are expected in the last few months of 2016.

\section{Metastatic melanoma therapy}

A plethora of trials involving new drugs for metastatic melanomas are underway, which is testimony to the great interest surrounding this pathology. A 2015 review described more than a hundred new trials [14] in the immunotherapy setting, involving checkpoint inhibitors, and other innovative treatment options.

The PDL-1 inhibitor atezolizumab is being studied in a Phase I trial, including its combination with vemurafenib or with vemurafenib plus cobimetinib [15]. Another PDL-1 inhibitor, MDX-1105, will soon be subject to clinical trials. An agonist of CD27, a critical regulator of $\mathrm{T}$ cells, called varlilumab, is being tested both in a Phase I study with ipilimumab and in a Phase II study [16]. The Phase II study includes the combination of varlilumab and ipilimumab versus varlilumab plus ipilimumab plus CDX-1401, a vaccine designed to activate the immune system against cancers that express the tumor marker NY-ESO-1, found in about $30 \%$ of melanomas. Another immunologic agonist is TRX-518, which binds the GITR (glucocorticoid-induced TNFR-related protein), a co-stimulatory factor to both $\mathrm{CD}^{+}{ }^{+}$- and $\mathrm{CD} 8^{+}$-naive cells, and enhances immune activity; a Phase I trial is underway and is estimated to be completed in 2016 [17].

A very interesting immunotherapeutic treatment is adoptive cell therapy. In this approach, tumor-infiltrating lymphocytes (TILs) are extracted, and then cultured and manipulated. For example, it is possible to make the lymphocytes express the CXCR 2 receptor. The only limitation of this approach is the timing, since TIL expansion takes about 5-6 weeks. Therefore, the approach can only be used in patients with an indolent melanoma. Furthermore, this could be a point of convergence between immunotherapy and biological therapy, since BRAF inhibitors such as vemurafenib seem to increase TIL expression. A Phase II study [18] is investigating the role of vemurafenib and IL-2 after the reinfusion of expanded TILs; results are expected in the second half of 2016.

Another interesting category of therapeutics is vaccines, which work by using cancer cell lysate tumor-associated antigens or linking the tumor-associated antigens to other molecules. However, the study exploring the activity of a vaccine from lysate cells, Mvax, in metastatic melanoma patients does not seem to be active anymore since results were expected in the beginning of 2015 [19]. 
Last, there are Phase III trials investigating the activity of an autologous dendritic cell-based treatment. Dendritic cells are one of the main triggers of immune-related responses since they can capture, elaborate and present the antigens to $T$ cells. Moreover, their presence in the microenvironment has been shown to be inversely proportional to tumor thickness and patient survival; the patient cells are expanded and loaded with autologous m-RNA, and then reinfused in the patient. A Phase III trial [20] compared a treatment based on cells suspended in GM-CSF versus cells suspended in GM-CSF mixed with irradiated tumor cells.

With regard to target therapy, the COLUMBUS trial, a study evaluating a new combination of BRAF and MEK inhibitors, LGX818 and MEK162, versus vemurafenib alone versus LGX 818 alone, is soon to close its enrollment and will present its first data in March 2018 [21]. Another promising BRAF inhibitor, PLX8394, is being studied in an ongoing Phase I/II trial [22].

The LOGIC 2 trial will test promising drugs that could avoid BRAF-MEK mechanisms of resistance in $B R A F$-mutated patients. This Phase II study investigates the efficacy, after progression, of adding one of four molecules to the standard of care dabrafenib and trametinib. The drug of choice depends on the melanoma genetic profile. The first molecule is Lee 011, a CDK4/6 inhibitor. The second one is BGJ398, a pan inhibitor of human FGFRs with potential antiangiogenic and antineoplastic activities. The third is BKM120 or buparlisib, a pan-FGFR kinase inhibitor that selectively binds to and blocks the activities of FGFRs, therefore inhibiting tumor angiogenesis and cell proliferation. The final molecule is INC280, a small c-met inhibitor [23].

Although there are a great number of new molecules, with a wide spectrum of clinical studies, it would also be interesting to understand how the already approved drugs could be combined for treatment or which drug is best suited to first-line treatment of $B R A F$-mutated melanomas [24]. A National Cancer Institute Phase III study, which has ipilimumab plus nivolumab followed by dabrafenib and trametinib compared with dabrafenib and trametinib followed by ipilimumab + nivolumab, could provide an answer to the question. First results are expected in April 2016 [25]. A Phase I/II study is trying to assess whether combining pembrolizumab with dabrafenib and trametinib could be safer and more effective than just the initial combination itself; its results are expected in late 2017 [26].

There is, however, no trial answering the following issues:

- How long should we administer immunotherapy?

- Could it be a good idea to switch from immunotherapy to biological therapy, or vice versa? In addition, when should that be done?

- Could it be feasible, in $B R A F$-mutated patients with long-term survival, to suspend the target therapy for a short period without a negative effect on the efficacy, in order to reduce adverse events?

In conclusion, while most of the studies involve new drugs, it could be helpful to have a small number of clinical trials investigating the best way to use the already available treatments. In this way, we could have an accurate algorithm and guidelines, helping both patients and practitioners, in few years.

\section{Financial \& competing interests disclosure}

The authors have no relevant affliations or financial involvement with any organization or entity with a financial interest in or financial conflict with the subject matter or materials discussed in the manuscript. This includes employment, consultancies, honoraria, stock ownership or options, expert testimony, grants or patents received or pending, or royalties.

No writing assistance was utilized in the production of this manuscript.

\section{References}

1 Kyi C, Postow MA. Checkpoint blocking antibodies in cancer immunotherapy FEBS. Lett. 588(2), 368-376 (2014).

2 Flaherty KT, Infanti JR, Audi A et al. Combine BRAF and MEK inhibition in melanoma with BRAF V600 mutations. N. Engl. J. Med. 367(18), 1694-1703 (2012).
3 Atkins MB, Kunkel L, Sznol M, Rosenberg SA. High-dose recombinant interleukin-2 therapy in patients with metastatic melanoma: long-term survival update. Cancer J. Sci. Am. 6(Suppl. 1), S11-S14 (2000).

4 Hodi FS, O’Day SJ, McDermott DF et al. Improved survival with ipilimumab in patients with metastatic melanoma. N. Engl. J. Med. 363(8), 711-723 (2010).

5 Robert C, Long GV, Brady B et al. Nivolumab in previously untreated melanoma without BRAF mutation. N. Engl. J. Med. 372, 320-330 (2015).

6 Robert C, Schachter J, Long GV et al. Pembrolizumab versus ipilimumab in 
advanced melanoma. N. Engl. J. Med. 372(26), 2521-2532 (2015).

7 Larkin J, Chiarion-Sileni V, Gonzalez R et al. Combined nivolumab and ipilimumab or monotherapy in untreated melanoma. N. Engl. J. Med.373(13), 1270-1271 (2015).

8 Chapman PB, Hauschild A, Robert C et al. Improved survival with vemurafenib in melanoma with BRAF V600E mutation. N. Engl. J. Med. 364, 2507-2516 (2011).

9 Hauschild A, Grob JJ, Demidov LV et al. Dabrafenib in $B R A F$-mutated metastatic melanoma: a multicentre, open-label, Phase 3 randomised controlled trial. Lancet 380(9839), 358-365 (2012).

10 Long GV, Stroyakovskiy D, Gogas $\mathrm{H}$ et al. Dabrafenib and trametinib versus dabrafenib and placebo for Val600 BRAF-mutant melanoma: a multicentre, double-blind, Phase 3 randomised controlled trial. Lancet 386(9992), 444-451 (2015).

11 Larkin J, Ascierto P, Dreno B et al. Combined vemurafenib and cobimetinib in $B R A F-$ mutated melanoma. N. Engl. J. Med. 371(20), 1867-1876 (2014).

12 Kirkwood JM,Strawderman MH, Ernstoff MS, Smith TJ, Borden EC, Blum RH. Interferon alfa-2b adjuvant therapy of high-risk resected cutaneous melanoma: the Eastern Cooperative Oncology Group Trial EST 1684. J. Clin. Oncol. 14(1), 7-17 (1996).
13 Eggermont AM, Chiarion-Sileni V, Grob JJ et al. Adjuvant ipilimumab versus placebo after complete resection of high-risk stage III melanoma (EORTC 18071): a randomised, double-blind, Phase 3 trial. Lancet. Oncol. 16(5), 522-530 (2015).

14 Rotte A, Bhandaru M, Zhou Y, McElwee KJ. Immunotherapy of melanoma: present options and future promises. Cancer Metastasis Rev. 34(1), 115-128 (2015).

15 A Phase 1b Study of Atezolizumab in Combination With Vemurafenib or Vemurafenib Plus Cobimetinib in Participants With BRAFV600-Mutation Positive Metastatic Melanoma. https://clinicaltrials.gov

16 A Study of Varlilumab (Anti-CD27) and Ipilimumab and CDX-1401 in Patients With Unresectable Stage III or IV Melanoma. https://clinicaltrials.gov

17 Trial of TRX518 (Anti-GITR mAb) in Stage III or IV Malignant Melanoma or Other Solid Tumors (TRX518-001). https://clinicaltrials.gov

18 Vemurafenib With Lymphodepletion Plus Adoptive Cell Transfer \& High Dose IL-2 Metastatic Melanoma. https://clinicaltrials.gov

19 M-Vax + Low Dose Interleukin-2 Versus Placebo Vaccine in Metastatic Melanoma in Patients With Stage IV Melanoma. https://clinicaltrials.gov
20 Autologous Dendritic Cell-Tumor Cell Immunotherapy for Metastatic Melanoma. https://clinicaltrials.gov

21 Study Comparing Combination of LGX818 Plus MEK162 Versus Vemurafenib and LGX818 Monotherapy in BRAF Mutant Melanoma (COLUMBUS). https://clinicaltrials.gov

22 As a Single Agent in Patients With Advanced, Unresectable Solid Tumors. https://clinicaltrials.gov

23 LGX818 and MEK162 in Combination With a Third Agent (BKM120, LEE011, BGJ398 or INC280) in Advanced BRAF Melanoma (LOGIC-2). https://clinicaltrials.gov

24 Ascierto PA, Grimaldi AM, Anderson AC et al. Future perspectives in melanoma research: meeting report from the "Melanoma Bridge", Napoli, December 5th-8th 2013. J. Transl. Med. 12, 277 (2014).

25 Dabrafenib and Trametinib Followed by Ipilimumab and Nivolumab or Ipilimumab and Nivolumab Followed by Dabrafenib and Trametinib in Treating Patients With Stage III-IV BRAFV600 Melanoma. https://clinicaltrials.gov

26 A Study of the Safety and Efficacy of Pembrolizumab (MK-3475) in Combination With Trametinib and Dabrafenib in Participants With Advanced Melanoma (MK-3475-3022/KEYNOTE-022). https://clinicaltrials.gov 